\title{
Instant soft robot: A simple recipe for quick and easy manufacturing
}

\author{
J. Fras ${ }^{1}$, J. Glowka ${ }^{2}$, K. Althoefer ${ }^{1}$
}

\begin{abstract}
Soft robots offer interesting features and can be manufactured cheaply using widely available lab equipment. However, with increasing robot complexity, the manufacturing process often becomes expensive, requires sophisticated or experimental tools, highly skilled technicians and time. In this paper, we propose an alternative approach for the fabrication of soft pneumatic fiber-reinforced actuators. With the new design, a scaffolding to reinforce the actuator is printed inside a singleuse mold. The mold is injected with silicone and broken apart, while the reinforcement detaches from the mold and remains within the actuator. Using flexible filaments such as nylon allows for the resulting actuators to be not only compliant but also squeezable. Our approach reduces the needed work effort and requires virtually no manufacturing skills when compared to current labor-intensive fabrication techniques. It also does not require any equipment other than an affordable, single nozzle desktop 3D printer. In the paper, we presented the design and fabrication process of different types of modules: linearly extending, bending and a manipulation module. We have compared the new instant design and the previous fiberreinforced design in terms of bending, elongation and generated forces.
\end{abstract}

\section{INTRODUCTION}

Soft robots are a promising alternative for traditional robotic devices. They are compliant, safe and offer outstanding motion capabilities. They are quickly gaining the interest of researchers around the world, and the features they offer are not the only reason. One of the reasons is soft robotics is fairly easy to experiment with. A good example are pneumatic soft robots. To start experimenting with them the only things needed are silicone material, 3D-printed molds, and some manual fabrication skills. Soft pneumatic robots can be actuated with a simple compressor or even a syringe. The problem is, the simplest silicone-based robots are very limited in terms of their motion capabilities and performance. One of the options to improve them is to add threads or fabrics to constrain the deformation of the silicone material and shape the robot structure in the desired way.

Non-extensible fibers embedded in the soft material allow for deformation only in the direction perpendicular to the fiber. Any other deformation in close proximity to the fiber is limited. There is a number of soft robot examples taking

*This work was supported in part by the EPSRC in the framework of the NCNR (National Centre for Nuclear Robotics) project (EP/R02572X/1) and the Innovate UK funded and q-bot led project WormBot (2308/104059).

${ }^{1} \mathrm{~J}$. Fras and K. Althoefer are with the Centre of Advanced Robotics @ Queen Mary (ARQ), Faculty of Science and Engineering, Queen Mary University of London, London E1 4NS, UK j. fras @ qmul.ac.uk, $\mathrm{k}$. althoefereqmul.ac.uk

${ }^{2} \mathrm{~J}$. Glowka is with the Industrial Research Institute for Automation and Measurements PIAP, Warsaw, PL jglowka@piap.pl

We would like to thank Yigit Menguc for his help in contextualizing the idea presented in this paper advantage of such a solution. In [1]-[4] fibers are used to preprogram the actuator behaviors, in particular enforce the deformation direction, its amount and constrain the final shape of the device. In [5] a non-stretchable mesh is used for constraining the deformation and [6] uses a fabric to constrain the deformation of flexible pneumatic cylinders allowing for high actuation pressures. In some cases an addition of rigid reinforcing structures allows for enhancing soft actuators performance [7].

The reinforcement can be added to the actuator at any stage of the manufacturing process. The simplest approach is to make the soft structure and then wrap it with fiber or fabric. This is, however, a very time demanding task. In many cases, since an inextensible structure (the thread) is applied to a soft surface (the silicone-made chamber wall), it is very likely to introduce unwanted stresses and tensions into the actuator body [8]. Another option is to incorporate the reinforcement inside the soft structure. In the case of cylindrically-shaped robots, this can be achieved by wrapping a thread around a rigid cylindrical rod, covering it with soft material, removing the rod and creating a soft layer inside the reinforcement. This way no stresses are induced, as the rod does not deform while being wrapped and the thread itself does not stretch even if some tension is required to wrap it around the rod. Such an approach helps to improve the reinforcement manufacturing, however, requires additional operations that are core wrapping and an extra molding step to create both sides (internal and external) of the actuator.

Any reinforcement embedded into the actuator adds to the manual work required to manufacture it. Moreover, due to the manufacturing constraints, most of the reinforcement methods proposed so far lead to relatively basic structures that constrain the deformation in a very simple way. Currently, in most cases, the reinforcement is made manually which results in poor repeatability.

An ideal solution would be to fabricate soft robot prototypes using additive methods such as $3 \mathrm{D}$ printing. There are technologies that allow printing with very stretchable materials [9]-[11], but parts with complex shapes and internal voids are still difficult to achieve with those approaches. In [12] a 3D printer modification allowing for printing with silicone material and reinforcing it with thin layers of filament have been proposed. The structures are printed on a rotating rod which limits the geometry to cylindrical. In [13], a printing technique combining various materials allowing for printing soft structures reinforced with fibers has been proposed.

The problem is all the aforementioned approaches rely on custom-made 3D-printers that are either not commercially available or very expensive, and, hence, making them inac- 
cessible for the wider soft robotics engineering community.

\section{A. Instant robot idea}

The goal of our research is to enable a fast and convenient way of prototyping soft pressure-driven robots that could be easily used by researchers and engineers. In our previous research, each design iteration required a set of molds to be printed and hours of manual work to fabricate the device. Any design adjustment was causing the molds to be redesigned and 3D printed again, then fiber reinforcement had to be prepared using dedicated rods. The rods wrapped with fibers were covered with silicone and then removed gently to not damage the fibers. The internal volumes were then filled with silicone to create internal actuator layers. Despite the hands-on experience and lots of design improvements, such a process is still very time-consuming. It is also very exclusive, as a non-experienced technician cannot easily reproduce the procedure without guidance.

In this paper, we propose a soft robot manufacturing approach that uses high-strain, high-elastic silicone materials and allows embedding reinforcement while keeping the amount of required manual work to a minimum. The idea uses disposable 3D-printed molds with embedded reinforcement structures. The molds and reinforcement structures are printed in one go and in a way that the reinforcement is over-molded and becomes a part of the actuator after the external mold is removed. The whole actuator mold can be printed with an affordable desktop FDM (Fused Deposition Modeling) 3D printer. The proposed approach has proven to provide similar motion performance when compared to fiberreinforced actuators while enabling faster manufacturing of prototypes and requiring much less manual work. It also potentially enables new reinforcing structures that cannot be achieved when following the manual fiber deposition or fabric incorporation approach.

A very important requirement for our design is to be printed using a single extruder FDM printer as those machines are the most popular and most affordable 3D printers.

Usage of soft filaments such as nylon allows not only compliant but also squeezable designs.

Since the main part of the fabrication process is to fill a 3D printed mold with silicone, wait and open it - just like instant food - we call our approach the "instant" soft robot.

\section{THE DESIGN}

The simplest possible implementation of the instant robot concept is a linear actuator. It would contain a cylindrical actuation chamber reinforced with circular rings of printed material. The actuator expands when pressurized, but the radial expansion is constrained by the reinforcing structures, so the only "deformation" is the elongation. The reinforcing rings are supported by teeth located at the internal side of the main mold. The reinforcement rings are printed not directly at the teeth, but slightly above them. Thanks to that they are still attached to the supporting structure, but the adhesion in between the mold and the reinforcement is weak at and breaks when the mold is being opened and torn away. Thus, the reinforcing rings are being separated from the mold and stay attached to the actuator. A similar approach is used for the removable support structures - they can be removed without damaging the actual printed object. The proper geometry of the supporting teeth and distance between them and reinforcement is a crucial aspect of the design.

The cross-section of a linear actuator design is presented in fig. 1.

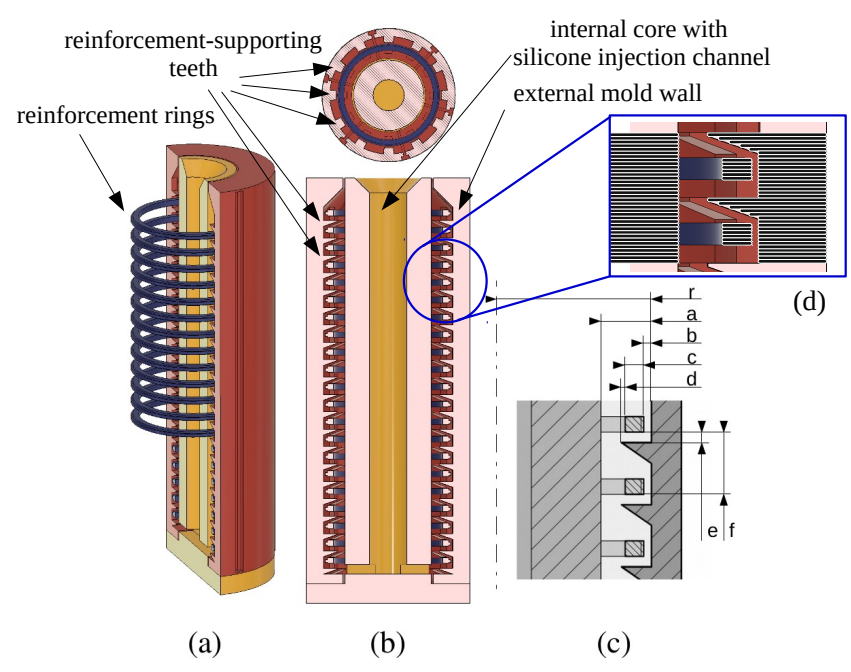

Fig. 1: Linear actuator mold cad model. The external wall in red, the internal core with silicone injection channel in yellow and reinforcement rings in blue. (a) and (b), Supporting teeth visible. Dimensions shown in (c) are as follows: $r=7.5 \mathrm{~mm}, a=2 \mathrm{~mm}, b=1.2 \mathrm{~mm}, c=0.8 \mathrm{~mm}, d=$ $0.4 \mathrm{~mm}, e=0.5 \mathrm{~mm}, f=1.8 \mathrm{~mm}$, (d) the design takes the layer height into account to have consistent number of layers and same geometry for each reinforcement period.

Adding a vertical continuum layer of 3D printed material connecting the reinforcement rings on one side of the linear actuator results in a strain-constraining structure, turning the linearly extending actuator into a bending one, fig. $2 \mathrm{a}$.

Connecting multiple actuation chambers in parallel leads to a multi-DOF (degrees of freedom) actuator. One of the possible implementations is a 3-DOF manipulation module as shown in fig. 2 .

The presented manipulation module design contains three identical actuation chambers reinforced with nylon structures. Each module is equipped with a hollow channel at the central axis so that pressure cables for subsequent modules can be passed through. The reinforcement is printed out of nylon material providing some flexibility so that the module can be squeezed without breaking, but it also introduces some rigidity, so that the pressure chamber does not deform much when pressurized despite the non-circular cross-section. This is an important aspect, as the deforming chamber would occlude the central channel and affect the pressure response making it less linear [8]. It would also speed up the wear and tear of the silicone material. However, 


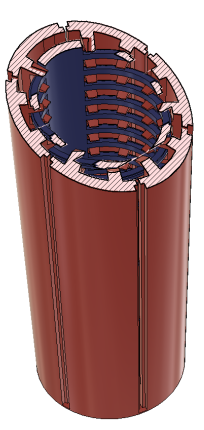

(a)

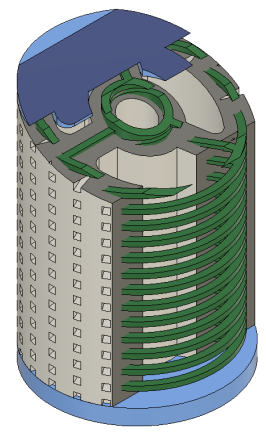

(b)

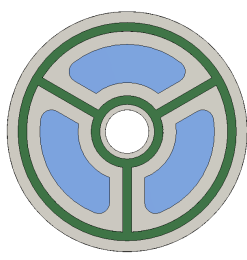

(c)
Fig. 2: Instant actuators design. (a) Bending actuator's mold, continuum layer of material exposed in blue (b) Manipulator side view, reinforcement exposed in green. Top and bottom sealing in blue. (b) Manipulator top view, actuation chambers (in blue) and central channel (in white) presented.

some deformation is still visible, resulting in a less linear response with regards to a module with circular chambers. Due to the process limitations, we are not able to embed circular reinforcement for each chamber, as the reinforcing structure has to be supported by the teeth located at the outer wall of the mold or overhung in the air. Overhangs can be only straight, so we ended up with a circle divided to sections, one section per actuation chamber. We considered a circular cross-section of the rods creating the actuation chambers, but such a modification would not prevent the cross-section from being deformed as the pressure transferred by the silicone would affect the geometry of the reinforcement anyway. Moreover, switching to circular chamber cross-section would reduce its actuation volume.

The manipulation module fabricated using the proposed approach is an interesting alternative for the STIFF-FLOP manipulator [3], [14] providing the same functionality and similar performance while offering a significantly reduced fabrication effort. STIFF-FLOP is a manipulator designed for minimal invasive surgery (MIS), having no rigid components [15]. It is pneumatic pressure-driven and uses no electricity making it MRI-compliant (Magnetic Resonance Imaging). One of the important STIFF-FLOP advantages is, it is cheap in production when compared to other MIS devices and utilizing no toxic materials making it an easy to use, disposable tool. With the proposed instant fabrication technique, such a device could become even cheaper allowing for use many of them during a single procedure with no significant increase of the procedure costs.

\section{FABRICATION}

\section{A. The mold}

As mentioned, the molds for the actuators fabrication are produced in a 3D printing process. We use an FDM technology printer which is a very popular and affordable tool. Our design is adjusted for a single nozzle machine, however, using more than one material in the same process would make things even simpler and would allow for more complex designs through the introduction of soluble supports.

The molds for our experiments were sliced using Prusa Slicer and printed with the Zortrax M200 machine [16]. We used soft Nylon PA12 filament. The printing parameters were adjusted to reduce the stringing effect, fig. 3 , and to ensure proper adhesion of the printed layers. The adhesion of layers composing the reinforcing structures has to be high enough to create a reliable reinforcing structure, but low enough to allow separation of the reinforcement when the mold is broken. Too strong adhesion between the outer mold and the reinforcement might break the structure that supposed to remain attached to the actuator body.

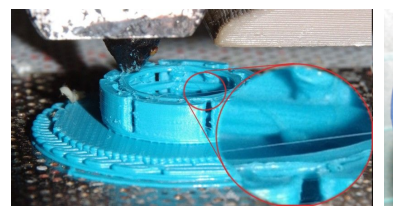

(a)

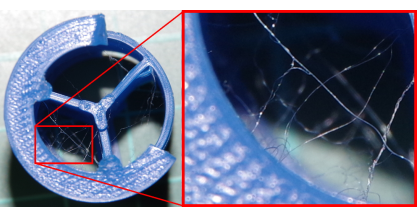

(b)
Fig. 3: (a) Mold being printed, (b) top-view. Some stringing effect visible.

The reinforcement separation problem could be improved by making the gap $e$ in fig. 1(c) bigger. On the other hand too big a gap $e$ might result in the filament not being attached to the supporting teeth at all and leading to a failed print.

Since the stringing effect is considerable, and in nearly all the prints we experienced some stringing, the design was split - the outer mold and the internal cores were printed separately. Thanks to that the mold can be blasted through with hot air, which efficiently removes all the strings and potential imperfections that would pierce the actuation chamber wall in the final actuator. Such a "small-feature removal" process has to be quick to not affect the actual structure of the mold. Another advantage of splitting the mold is that the internal cores are not destroyed in the demolding process and can be reused.

The main disposable mold of the manipulator module and the internal cores that can be reused are presented in fig. 4

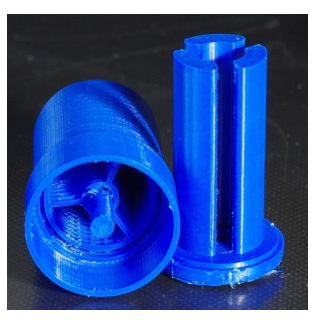

(a)

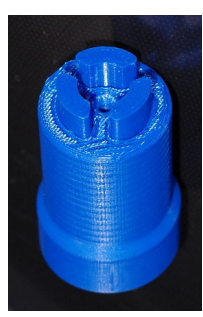

(b)

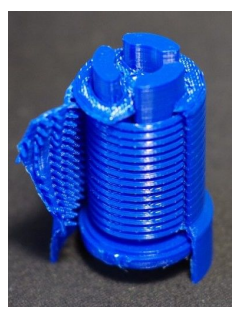

(c)
Fig. 4: 3D printed mold. (a) Mold parts - external single-use part and internal reusable rods. (b) Mold assembled, ready to be filled with silicone, (c), mold broken, internals shown. 


\section{B. The actuator}

After the mold is cleaned with hot air, it is put together (the rods are inserted into the external mold) and it is filled with silicone by injecting the material through the injection channel. For this step an ordinary syringe is used, no special equipment required.

The internal injection channel ensures the silicone material fills the mold from the bottom up helping to remove all the air from the mold efficiently. Thanks to that the silicone body is consistent and does not contain any air bubbles trapped during molding. (It is noted that the silicone material has to be properly degassed before molding commences.) After the silicone is cured, the external mold is broken apart and the internal rods are pulled out. The reinforcing rings get separated from the outer mold during this process and remain embedded into the actuator. The actuator is then sealed on both ends with caps molded in another fabrication step. The whole procedure is presented in fig. 5 .

(a)

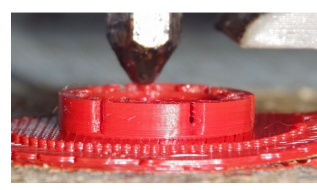

(c)

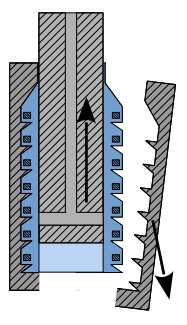

(e)
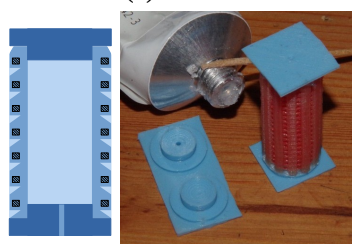

Fig. 5: Actuator manufacturing. The mold is printed using FDM printer (a), then assembled and injected with the uncured silicone material (b). After silicone cures the mold is broken apart and removed (c) while the reinforcement remains attached to the actuator's body (d). Then top and bottom caps are attached (e). In (f) the ready-to-action actuator is presented.

Finished linear and bending actuators, as well as a manipulation module, are presented in fig. 6 .

\section{INSTANT SOFT ROBOT}

The manipulation modules can be stacked together creating a soft robotic arm.

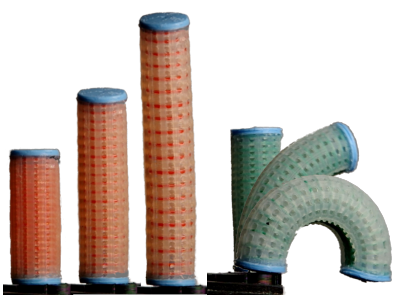

(a) (b)

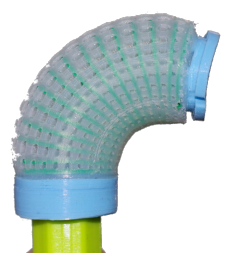

(c)
Fig. 6: Instant actuators, silicone used SmoothOn Ecoflex 0050 [17], (a) linear expanding actuator, (b) bending actuator, (c) manipulation module

When compared to the previous manufacturing process of the STIFF-FLOP manipulator, we got rid of the most time-consuming and most skill-demanding step - the reinforcement fabrication. Such a step was based on winding cylindrical rods with a polyester thread. The cores were composed of 3 parts and were wrapped with the thread manually using a drill. There were 6 identical cores per module required. We also reduced the number of molding steps. The reduction of the number of molding iterations not only reduces the amount of manual work but further contributes to the reduction of the overall fabrication time due to the silicone curing time. It is noted that the procedure described in [14] has already been optimized towards easy, quick and reliable manufacturing, other procedures proposed for similar actuators are often more labor-intensive [8].

The STIFF-FLOP manipulator and the new instant manipulator are presented in fig. 7.

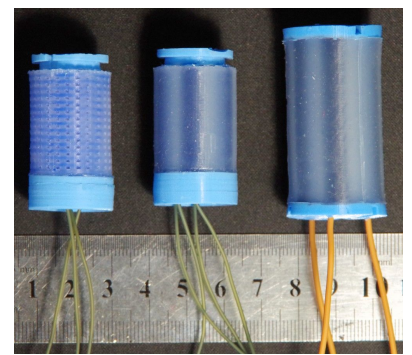

(a)

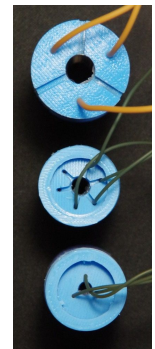

(b)

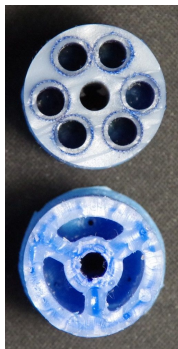

(c)
Fig. 7: (a) instant manipulator (left), STIFF-FLOP-like fiberreinforced reference (middle) and the actual STIFF-FLOP manipulator (right) modules compared, (b) bottom view, (c) top view, cross-section.

In parallel to introducing improvements in manufacturing, we have also advanced the design with regards to the ease of use of the modules. The STIFF-FLOP manipulator was originally assembled by gluing on attachment rings that were then screwed together. Disassembly operations in case any module needed replacement was time-consuming. Moreover, the attachment rings were rigid, making the robot notperfectly soft across the entire structure and compromising some of the possible applications. In the new design, we equipped the manipulation modules with a base and tip hav- 


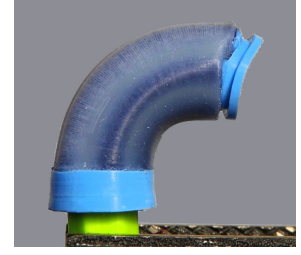

(a)

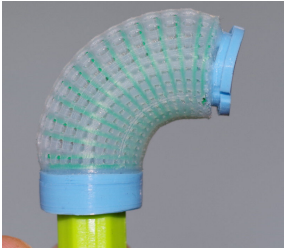

(b)
Fig. 8: Actuation of (a) fiber-reinforced and (b) instant modules.

ing a geometry that allows for form-locking the segment ends into each other. This makes the assembly and disassembly process very easy and straightforward, fig. 9. The assembled 3-module manipulator is presented in fig. 10.

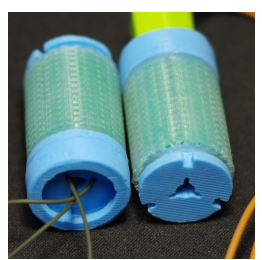

(a)

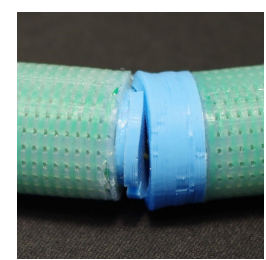

(b)
Fig. 9: Shape locking structure, connection mechanism

The length of a single instant manipulator module is $38 \mathrm{~mm}$ while its diameter is $22 \mathrm{~mm}$. The central channel width is $4.5 \mathrm{~mm}$

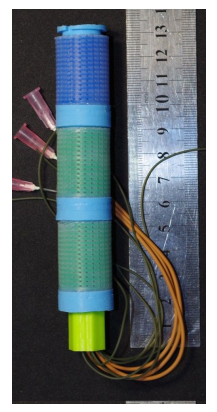

(a)

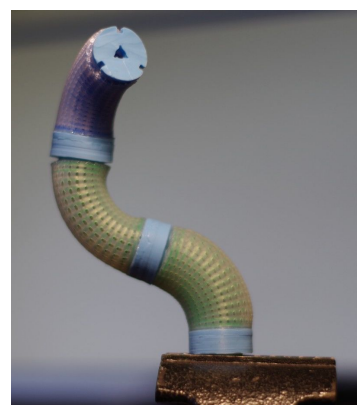

(b)
Fig. 10: Instant manipulator assembled.

\section{EXPERIMENTS}

The proposed actuators and the manipulator module were tested in terms of generated motions and forces. For the deformation detection, we used a vision-based system equipped with a video camera. Color markers were attached to the actuators and tracked with an image processing algorithm. For the force measurement, we used the Robotous RFT40SA01 force sensor. In our tests, the pressure was controlled with SMC ITV0050 pressure regulators.

We have measured the bending characteristics of the manipulation module as well as the force generated by the manipulation module in a parallel and perpendicular direction to its main axis with one, two and three chambers actuated. To prove the concept a reference STIFF-FLOPlike manipulation module has been fabricated. The external dimension of the instant and the fiber-reinforced modules match so that they can be easily swapped in the final setup. The cross-section area of actuation chambers is equal in both designs. The measurement setup is presented in fig. 11.

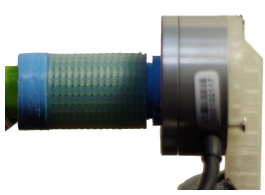

(a)

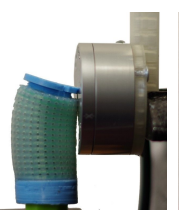

(b)

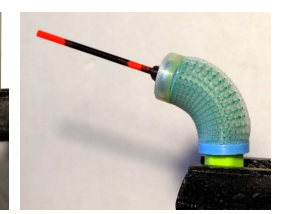

(c)
Fig. 11: Measurement setup. (a) force parallel and (b) perpendicular to the main axis of the manipulation module, (c) bending and elongation characteristics setup.

Initial tests with modules show that the instant actuator is significantly less stiff than the reference fiber-reinforced module made of the same material. This is related to the fact that the instant actuator contains less silicone material on its outer side due to the reinforcement supporting teeth and the bigger volume of the reinforcement itself. The reinforcement material detaches from the silicone wall while the actuator body deforms, making the resulting cross-section inertia smaller. Effectively the pressure required to actuate the device is reduced. The bending characteristics of both, the fiber-reinforced and instant manipulation modules as well as linear actuators are presented in fig. 12 and fig. 13.

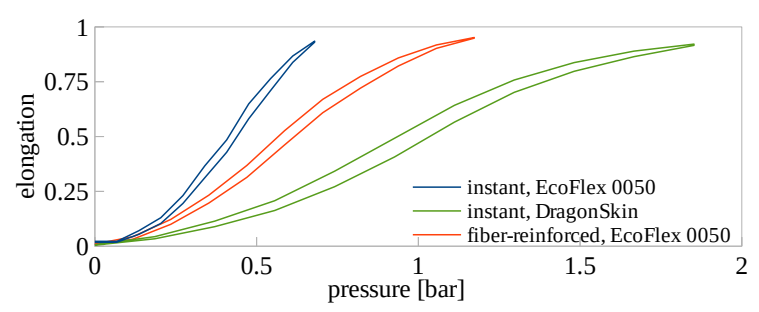

Fig. 12: Characteristics of instant and fiber-reinforced linear actuators.

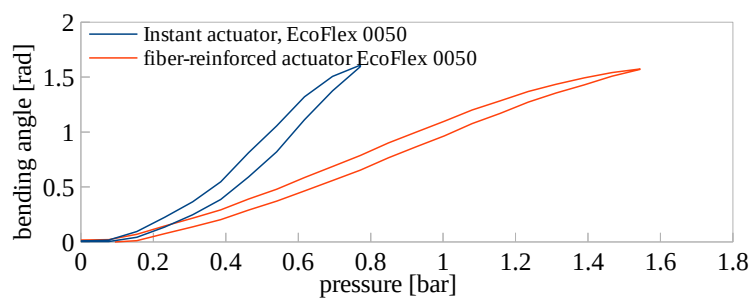

Fig. 13: Bending characteristics of instant and fiberreinforced manipulation modules.

For that reason, we decided to manufacture the proposed manipulation actuator with a stiffer Dragon Skin 10 silicone [18], which moved the characteristics considerably closer to the reference device. The gathered data for the instant 
actuator (Dragon Skin 10 material) and the fiber-reinforced reference design (EcoFlex 0050 material) is presented in figures 16,15 and 14 .
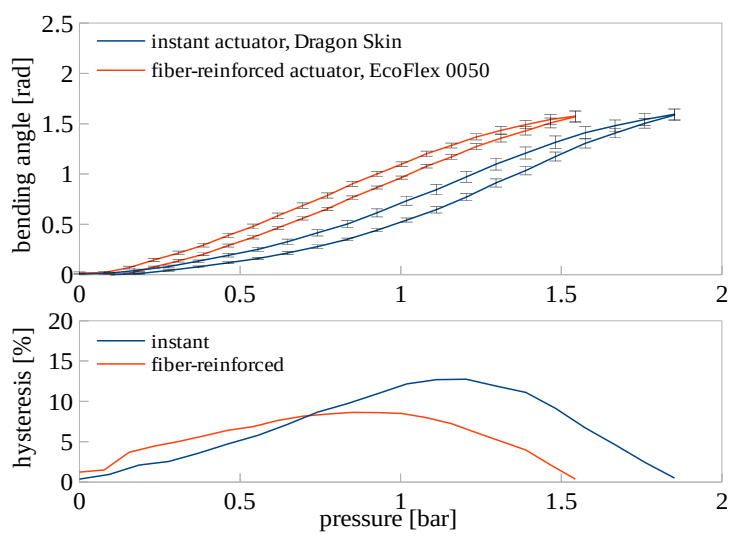

(a)
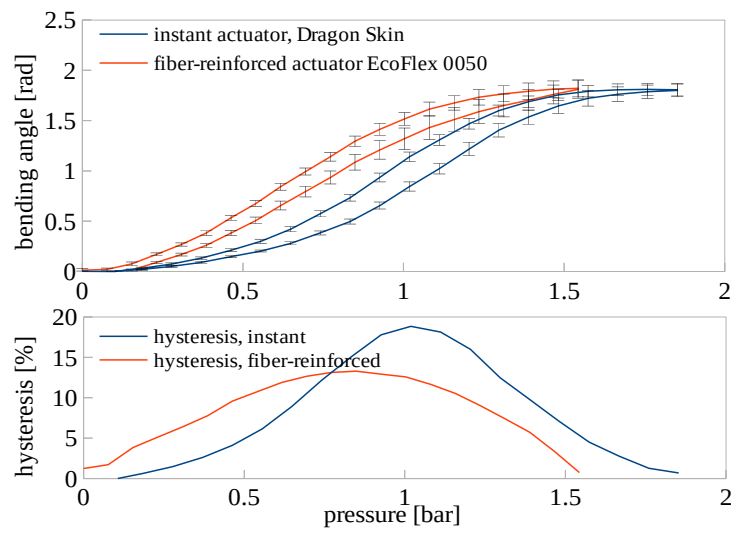

(b)

Fig. 14: The bending characteristics of the manipulator, fig. 11c single chamber (a) and two chambers activated (b). Measurement taken for each chamber three times, 9 measurements in total per module.

Above a certain pressure value, the compressed gas in the instant manipulation module tends to push the reinforcement away, fig. 17a-c. Such a displacement creates areas that tend to balloon. Ballooning, in turn, leads to bursts. Destructive tests show the bursts occur at approximately 2.4 bar pressure at approximately 2.4 radians actuation angle for the instant actuator while the reinforcement started failing earlier, at around 2 bars. We were not able to explode the fiberreinforced design within the pressure we control with our hardware ( 3 bars, more than 3 radians of bending), fig. 17. In fig. 18 the destructive tests data is presented.

\section{DISCUSSION}

The instant actuator having the same external dimensions and equal actuation area to a reference fiber-reinforced actuator requires significantly lower pressure to be actuated. This is related to a reduced amount of silicone material contributing to the outer part of its body due to wholes created by reinforcement-supporting teeth and the volume of

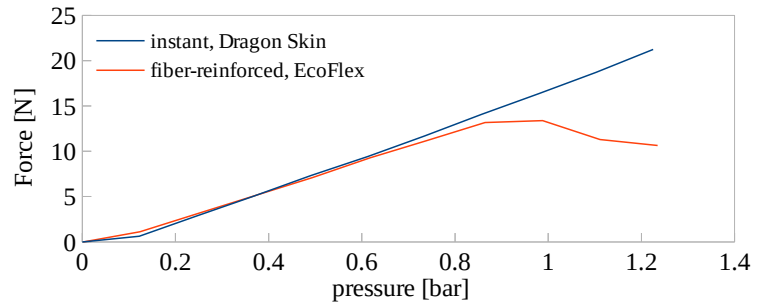

Fig. 15: The force generated by the manipulator along its primary axis, fig. $11 \mathrm{a}$. The reference manipulator started to buckle around 0.8 bar.

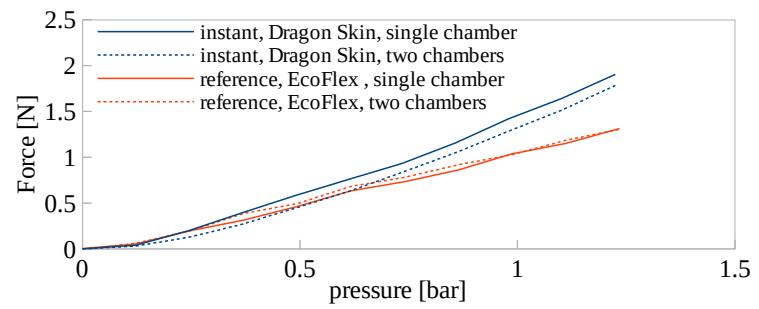

Fig. 16: The force generated by the manipulator perpendicular to its main axis, fig. $11 \mathrm{~b}$

the reinforcement itself. The stiffness of the instant actuator is reduced due to the same reason. Such an effect can be compensated by using a stiffer material for fabrication of the instant actuator.

The instant manipulation module behavior is less linear than the reference one, this is probably related to non-circular pneumatic chamber cross-sections in the instant version. Due to that, the geometry of the chamber can change resulting in the actuation area growth. Such a change does not occur in cylindrical chambers of the reference manipulator making it much more linear.

The deformation of the actuation chambers and the semiflexible-semi-rigid reinforcement also affects the hysteresis making it slightly bigger.

It is also noted that due to the reduced density of the reinforcement the instant actuator fails at a smaller actuation angle than the dense reinforced reference module. Still, the instant actuator can easily operate up to 90 degrees angle which makes it a good choice for a variety of applications. The reinforcing structures themselves never failed in our tests, the bursts always occur due to their displacement and ballooning of the silicone body between the reinforcement.

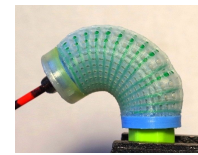

(a)

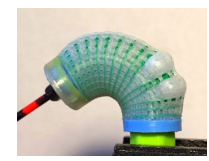

(b)

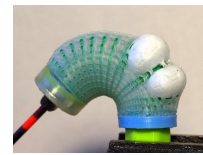

(c)

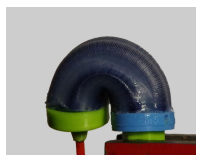

(d)
Fig. 17: Ballooning between reinforcement rings occurring above a certain pressure. (a) at approximately 2 bar the reinforcement starts to displace, (c) at 2.4 the actuator bursts, (d) reference actuator actuated to $\pi$ radians with no harm. 


\begin{tabular}{|c|c|c|c|c|}
\hline & STIFF-FLOP & instant manipulator & linear actuator & instant linear actuator \\
\hline required labour time & $\approx 3 \mathrm{~h}$ & $\approx 50 \mathrm{~m}$ & $\approx 1 \mathrm{~h}$ & $\approx 40 \mathrm{~m}$ \\
\hline skills/ experience & high & low & medium & low \\
\hline mold & 34 parts, reusable & 6 pats (5 reusable) & 10 parts, reusable & 4 parts (3 reusable) \\
\hline molding steps & 3 & 2 & 3 & 2 \\
\hline gluing steps & 1 & 1 & 1 & 1 \\
\hline printing time & $7 \mathrm{~h} 30 \mathrm{~m}$ & $2 \mathrm{~h} 40 \mathrm{~m}$ once $\& 1 \mathrm{~h} 20 \mathrm{~m}$ per module & $3 \mathrm{~h} 35 \mathrm{~m}$ & $2 \mathrm{~h}$ once $\& 1 \mathrm{~h} 20 \mathrm{~m}$ per actuator \\
\hline
\end{tabular}

TABLE I: Manufacturing comparison, instant vs fiber-reinforced actuators

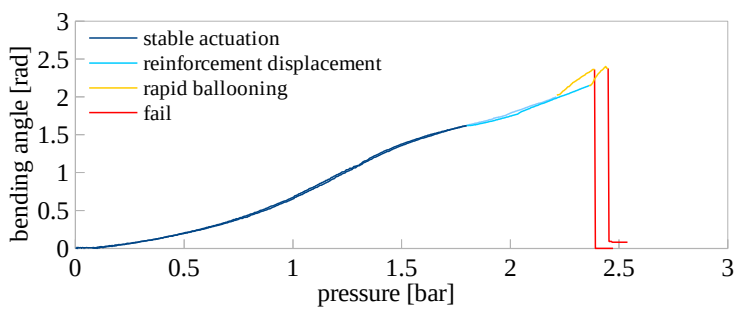

Fig. 18: Destructive tests of the manipulation module. Two chambers tested, third one got damaged while testing two first ones. Thanks to the automated reinforcement fabrication the charactersitics of both chambers is almost perfectly aligned in the stable operational range.

However, we were able to break the reinforcement by squeezing it in a repetitive manner for many times.

The actuation properties of the instant linear actuator are very similar to the fiber-reinforced one. Except the quantitative difference in the pressure required to achieve an arbitrary elongation, the linearity of the response and the hysteresis are comparable.

\section{CONCLUSION}

In this paper, we presented a new approach for the fabrication of fiber-reinforced actuators. Our work extends from the standard fabrication process of fiber-reinforced actuators automating most parts of the whole process reducing the required fabrication labor and needed manual skills to a minimum, see table I. The actuation performance of the actuators is comparable to that of previous fiber-reinforced actuators.

It was important for us to make the fabrication and use of soft material actuators accessible to soft robotic enthusiasts and researchers who may not have access to specialized equipment. There are advanced 3D printing alternatives offering to print soft materials directly or even mixing stretchable and not-stretchable materials in one process but due to their price or early stage of development, they are very exclusive and not available to everyone in the community. Using flexible printing materials such as nylon allows for the fabricated actuators to not only be compliant but also squeezable making the device entirely soft with no rigid components.

To prove our approach we have successfully designed and fabricated a soft pneumatic linear actuator, a bending actuator, and a 3-DOF manipulation module. The manipulation modules can be stacked one on top of another to make a modular, entirely soft manipulator.
The designs of the manipulator module and both, linear and bending actuators are available online at https:// github.com/jfras/instant_soft_robotics.

\section{REFERENCES}

[1] Fionnuala Connolly, Panagiotis Polygerinos, Conor J. Walsh, and Katia Bertoldi. Mechanical programming of soft actuators by varying fiber angle. Soft Robotics, 2(1):26-32, March 20152015.

[2] Kevin C Galloway, Panagiotis Polygerinos, Conor J Walsh, and Robert J Wood. Mechanically programmable bend radius for fiberreinforced soft actuators. In Advanced Robotics (ICAR), 2013 16th International Conference on, pages 1-6. IEEE, 2013.

[3] J. Fras, J. Czarnowski, M. Macias, J. Glowka, M. Cianchetti, and A. Menciassi. New stiff-flop module construction idea for improved actuation and sensing. In International Conference on Robotics and Automation, pages 2901-2906. IEEE, 2015.

[4] J. Fras, Y. Noh, H Wurdemann, and K. Althoefer. Soft fluidic rotary actuator with improved actuation properties. In International Conference on Intelligent Robots and Systems. IEEE, 2017.

[5] J. H. Pikul, S. Li, H. Bai, R. T. Hanlon, I. Cohen, and R. F. Shepherd. Stretchable surfaces with programmable $3 \mathrm{~d}$ texture morphing for synthetic camouflaging skins. Science, 358(6360):210-214, 2017.

[6] A. Stilli, H. A Wurdemann, and K. Althoefer. A novel concept for safe, stiffness-controllable robot links. Soft Robotics.

[7] Wookeun Park, Seongmin Seo, and Joonbum Bae. A hybrid gripper with soft material and rigid structures. IEEE Robotics and Automation Letters, 2018.

[8] Jan Fras and Kaspar Althoefer. Soft fiber-reinforced pneumatic actuator design and fabrication: Towards robust, soft robotic systems. In Towards Autonomous Robotic Systems. Springer International Publishing, 2019.

[9] TJ Wallin, J Pikul, and RF Shepherd. 3d printing of soft robotic systems. Nature Reviews Materials, page 1, 2018.

[10] S. Walker, O.D. Yirmibeşoğlu, U. Daalkhaijav, and Y. Mengüç. 14 - additive manufacturing of soft robots. In Robotic Systems and Autonomous Platforms, pages 335 - 359. Woodhead Publishing, 2019.

[11] Bryan N Peele, Thomas J Wallin, Huichan Zhao, and Robert F Shepherd. 3d printing antagonistic systems of artificial muscle using projection stereolithography. Bioinspiration \& biomimetics, 10(5):055003, 2015.

[12] Oisín Byrne, Fergal Coulter, Mark Glynn, James F.X. Jones, Aisling Ní Annaidh, Eoin D. O'Cearbhaill, and Dónal P. Holland. Additive manufacture of composite soft pneumatic actuators. Soft Robotics, 5(6):726-736, 2018. PMID: 30148682.

[13] M. Schaffner, J. Faber, L. Pianegonda, et al. 3d printing of robotic soft actuators with programmable bioinspired architectures. Nature Communications, 9, 122018.

[14] J. Fras, M. Macias, J. Czarnowski, et al. Soft manipulator actuation module-with reinforced chambers. Soft and Stiffness-controllable Robotics Solutions for Minimally Invasive Surgery: The STIFF-FLOP Approach, page 47, 2018.

[15] A. Arezzo, Y. Mintz, M. E. Allaix, S. Arolfo, M. Bonino, G. Gerboni, M. Brancadoro, et al. Total mesorectal excision using a soft and flexible robotic arm: a feasibility study in cadaver models. Surgical Endoscopy, pages 1-10, 2016.

[16] Zortrax m200 product page. https://zortrax.com/pl/ 3d-printers/m200/. Accessed: 2019-11-08.

[17] Ecoflex 00-50 product page. https://www.smooth-on.com/ products/ecoflex-00-50/. Accessed: 2017-09-15.

[18] Dragonskin product page. https://www.smooth-on.com/ products/dragon-skin-10-fast/. Accessed: 2017-09-15. 